\title{
Parallel Reducibility for Information-Theoretically Secure Computation
}

\author{
Yevgeniy Dodis ${ }^{1}$ and Silvio Micali ${ }^{1}$ \\ Laboratory for Computer Science, Massachusetts Institute of Technology, USA. \\ \{yevgen, silvio\}@theory.lcs.mit.edu
}

\begin{abstract}
Secure Function Evaluation (SFE) protocols are very hard to design, and reducibility has been recognized as a highly desirable property of SFE protocols. Informally speaking, reducibility (sometimes called modular composition) is the automatic ability to break up the design of complex SFE protocols into several simpler, individually secure components. Despite much effort, only the most basic type of reducibility, sequential reducibility (where only a single sub-protocol can be run at a time), has been considered and proven to hold for a specific class of SFE protocols. Unfortunately, sequential reducibility does not allow one to save on the number of rounds (often the most expensive resource in a distributed setting), and achieving more general notions is not easy (indeed, certain SFE notions provably enjoy sequential reducibility, but fail to enjoy more general ones).
\end{abstract}

In this paper, for information-theoretic SFE protocols, we

- Formalize the notion of parallel reducibility, where sub-protocols can be run at the same time;

- Clarify that there are two distinct forms of parallel reducibility:

* Concurrent reducibility, which applies when the order of the subprotocol calls is not important (and which reduces the round complexity dramatically as compared to sequential reducibility); and

* Synchronous reducibility, which applies when the sub-protocols must be executed simultaneously (and which allows modular design in settings where sequential reducibility does not even apply).

- Show that a large class of SFE protocols (i.e., those satisfying a slight modification of the original definition of Micali and Rogaway [15]) provably enjoy (both forms of) parallel reducibility.

\section{Introduction}

The objective of this paper is to understand, define, and prove the implementability of the notion of parallel reducibility for information-theoretically secure multiparty computation. Let us start by discussing the relevant concepts.

M. Bellare (Ed.): CRYPTO 2000, LNCS 1880, pp. 74-92 2000.

(C) Springer-Verlag Berlin Heidelberg 2000 
SFE Protocols. A secure function evaluation (SFE) is a communication protocol enabling a network of players (say, having a specified threshold of honest players) to compute a (probabilistic) function in a way that is as correct and as private as if an uncorruptable third party had carried out the computation on the players' behalf. SFE protocols were introduced by Goldreich, Micali and Wigderson [13] in a computational setting (where the parties are computationally bounded, but can observe all communication), and by Ben-Or, Goldwasser and Wigderson [4] and Chaum, Crépeau and Damgård [7] in an informationtheoretic setting (where the security is unconditional, and is achieved by means of private channel@). We focus on the latter setting.

SFE Definitions. Together with better SFE protocols, increasingly precise definitions for information-theoretic SFE have been proposed; in particular, those of Beaver [2], Goldwasser and Levin [11], Canetti [5], and Micali and Rogaway [15]. At a high-level, these definitions express that whatever an adversary can do in the real model (i.e., in the running of the actual protocol, where no trusted party exists) equals what an adversary can do in the ideal model (i.e., when players give their inputs to the trusted third party, who then computes the function for them). This more or less means that the most harm the adversary can do in the real model consists of changing the inputs of the faulty players (but not based on the inputs of the honest players!), and then running the protocol honestly.

All these prior definitions are adequate, in the sense that they (1) reasonably capture the desired intuition of SFE, and (2) provide for the existence of SFE protocols (in particular, the general protocol of [4] satisfies all of them). Were properties (1) and (2) all one cared about, then the most "liberal" definition of SFE might be preferable, because it would allow a greater number of reasonable protocols to be called secure. However, if one cared about satisfying additional properties, such as reducibility (i.e., as discussed below, the ability of designing SFE protocols in a modular fashion), then more stringent notions of SFE would be needed.

Reducibility and Sequential Reducibility. Assume that we have designed a SFE protocol, $F$, for a function $f$ in a so called semi-ideal model, where one can use a trusted party to evaluate some other functions $g^{1}, \ldots, g^{k}$. Assume also that we have designed a SFE protocol, $G_{i}$, for each function $g^{i}$. The reducibility property says that, by substituting the ideal calls to the $g^{i}$ 's in $F$ with the corresponding SFE protocols $G_{i}$ 's, we are guaranteed to obtain a SFE protocol for $f$ in the real model.

Clearly, reducibility is quite a fundamental and desirable property to have, because it allows one to break the task of designing a secure protocol for a complex function into the task of designing secure protocols for simpler functions. Reducibility, however, is not trivial to satisfy. After considerable effort, only the the most basic notion of reducibility, sequential reducibility, has been proved

$\overline{1}$ This means that every pair of players has a dedicated channel for communication, which the adversary can listen to only by corrupting one of the players. 
to hold for some SFE notions: those of [5] and [15]. Informally, sequential reducibility guarantees that substituting the ideal calls to the $g^{i}$ 's in $F$ with the corresponding $G_{i}$ 's yields a SFE protocol for $f$ in the real model only if a single $G_{i}$ is executed (in its entirety!) at a time Therefore, sequential reducibility is not general enough to handle protocols like the expected $O(1)$-round Byzantine agreement protocol of [10] (which relies on the concurrent execution of $n^{2}$ specific SFE protocols) whose security, up to now, must be proven "from scratch".

\subsection{Our Results}

In this paper, we put forward the notion of parallel reducibility and show which kinds of SFE protocols satisfy it. We actually distinguish two forms of parallel reducibility:

- Concurrent reducibility.

This type of reducibility applies when, in the semi-ideal model, the $g^{1}, \ldots, g^{k}$ can be executed in any order. The goal of concurrent reducibility is improving the round-complexity of modularly designed SFE protocols.

- Synchronous reducibility.

This type of reducibility applies when, in the semi-ideal model, the $g^{1}, \ldots, g^{k}$ must be executed "simultaneously." The goal of synchronous reducibility is enlarging the class of modularly designed SFE protocols (while being roundefficient as well).

Concurrent Reducibility. There are many ways to schedule the execution of several programs $G_{1}, \ldots, G_{k}$. Each such way is called an interleaving. The $k$ ! sequential executions of $G_{1}, \ldots, G_{k}$ are examples of interleavings. But they are very special and "very few," because interleavings may occur at a round-level. For instance, we could execute the $G_{i}$ 's one round at a time in a round-robin manner, or we could simultaneously execute, in single round $r$, the $r$-th round (if any) of all the $G_{i}$ 's. Saying that programs $G_{1}, \ldots, G_{k}$ are concurrently executable, relative to some specified goal, means that this goal is achieved for all of their interleavings.

Assume now that a function $f$ is securely evaluated by a semi-ideal protocol $F$ which, in a set of contiguous instructions, only makes ideal calls to functions $g^{1}, \ldots, g^{k}$, and let $G_{i}$ be a SFE protocol for $g^{i}$ (in the real model). Then, a fundamental question arise:

Will substituting each $g^{i}$ with $G_{i}$ yield a (real-model) SFE protocol for $f$ in which the $G_{i}$ 's are concurrently executable?

Let us elaborate on this question. Assume, for instance, that $F$ calls $g^{2}$ on inputs that include an output of $g^{1}$. Then we clearly cannot hope that the $G_{i}$ 's are

$\overline{2}$ This is true even if, within $F$, one could "ideally evaluate" all or many of the $g^{i}$ s "in parallel." 
concurrently executable. Thus, to make sense of the question, all the inputs to the $g^{i}$ 's should be determined before any of them is ideally evaluated. Moreover, even if all the $g^{i}$ 's are evaluated on completely unrelated and "independent" inputs, $F$ may be secure only for some orders of the $g^{i}$ 's, but not for others, which is illustrated by the following example.

Example 1: Let $f$ be the coin-flipping function (that takes no inputs and outputs a joint random bit), let $g^{1}$ be the coin-flipping function as well, and let $g^{2}$ be the majority function on $n$ bits. Let now $F$ be the following semi-ideal protocol. Each player $P_{j}$ locally flips a random bit $b_{j}$. Then the players "concurrently" use ideal calls to $g^{1}$ and $g^{2}\left(b_{1}, \ldots, b_{n}\right)$, getting answers $r$ and $c$ respectively. The common output of $F$ is $r \oplus c$. We claim that $F$ is secure if we first call $g^{2}$ (the majority) and then $g^{1}$ (the coin-flip), but insecure if we do it the other way around. Indeed, irrespective of which $c$ we get in the first ordering, since $r$ is random (and independent of $c$ ), then so is $r \oplus c$. On the other hand, assume we first learn the random bit $r$ and assume faulty players want to bias the resulting coin-flip towards 0 . Then the faulty players pretend that their (supposedly random) inputs $b_{j}$ for the majority are all equal to $r$. Provided there are enough faulty players, this strategy will bias the outcome $c$ of $g^{2}$ (the majority) towards $r$, and thus the output of $F$ towards 0 .

Clearly, in the case of the above example, we cannot hope to execute the $G_{i}$ 's concurrently: one of the possible interleavings is the one that sequentially executes the $G_{i}$ 's in the order that is insecure even in the semi-ideal model. Thus, the example illustrates that the following condition is necessary for the concurrent execution of the $G_{i}$ 's.

Condition 1: $F$ is secure in the semi-ideal model for any order of the $g^{i}$ s.

Is this necessary condition also sufficient? Of course, the answer also depends on the type of SFE notion we are using. But, if the answer were YES, then we would get the "strongest possible form of concurrent reducibility." Let us then be optimistic and put forward the following informal definition.

Definition 1: We say that a SFE notion satisfies concurrent reducibility if, whenever the protocols $F, G_{1}, \ldots, G_{k}$ satisfy this SFE notion, Condition 1 is (both necessary and) sufficient for the concurrent execution of the $G_{i}$ 's inside $F$ (in the real model).

Our optimism is justified in view of the following

Theorem 1: A slight modification of the SFE notion of Micali and Rogaway [15] satisfies concurrent reducibility.

We note that the SFE notion of Micali and Rogaway is the strictest one proposed so far, and that we have been unable to prove analogous theorems for all other, more liberal notions of SFE. We conjecture that no such analogous theorems exist for those latter notions. In support of our conjecture, we shall point out in 
Section 4.3 the strict properties of the definition of [15] that seem to be essential in establishing Theorem 1.

We remark that concurrent reducibility is important because it implies significant efficiency gains in the round-complexity (often the most expensive resource) of modularly designed SFE protocols. This is expressed by the following immediate Corollary of Definition 1.

Corollary 1: Assume that $F, g^{1}, \ldots, g^{k}$ satisfy Condition 1 , that $G_{i}$ is a protocol for $g^{i}$ taking $R_{i}$ rounds, and that $F, G_{1}, \ldots, G_{k}$ are SFE protocols according to a SFE notion satisfying concurrent reducibility. Then, there is a (real model) SFE implementation of $F$ executing all the $G_{i}$ 's in $\max \left(R_{1}, \ldots, R_{k}\right)$ rounds.

This number of rounds is the smallest one can hope for, and should be contrasted with $R_{1}+\cdots+R_{k}$, the number of rounds required by sequential reducibility.

Synchronous Reducibility. The need to execute several protocols in parallel does not necessarily arise from efficiency considerations or from the fact that it is nice not to worry about the order of the execution. A special type of parallel execution, synchronous execution, is needed for correctness itself.

Example 2: Let $f$ be the two-player coin-flipping function that returns a random bit to the first two players, $P_{1}$ and $P_{2}$, of a possibly larger network. That is, $f(\lambda, \lambda, \lambda, \ldots, \lambda)=(x, x, \lambda, \ldots, \lambda)$, where $x$ is a random bit (and $\lambda$ is the empty string). Consider now the following protocol $F$ : player $P_{1}$ randomly and secretly selects a bit $x_{1}$, player $P_{2}$ randomly and secretly selects a bit $x_{2}$, and then $P_{1}$ and $P_{2}$ "exchange" their selected bits and both output $x=x_{1} \oplus x_{2}$.

Clearly, $F$ is a secure function evaluation of $f$ only if the exchange of $x_{1}$ and $x_{2}$ is "simultaneous", that is, whenever $P_{1}$ learns $x_{2}$ only after it declares $x_{1}$, and vice versa. This requirement can be modeled as the parallel composition of two sending protocols: $g^{1}\left(x_{1}, \lambda, \lambda, \ldots, \lambda\right)=\left(x_{1}, x_{1}, \lambda, \ldots, \lambda\right)$ and $g^{2}\left(\lambda, x_{2}, \lambda, \ldots, \lambda\right)=$ $\left(x_{2}, x_{2}, \lambda, \ldots, \lambda\right)$. That is, we can envisage a semi-ideal protocol in which players $P_{1}$ and $P_{2}$ locally flip coins $x_{1}$ and $x_{2}$, then simultaneously evaluate $g^{1}$ and $g^{2}$, and finally exclusive OR their outputs of $g^{1}$ and $g^{2}$. However, no sequential order of the ideal calls to $g^{1}$ and $g^{2}$ would result in a secure two-player coin-flipping protocol. This example motivates the introduction of a special type of parallel composition (for security rather than efficiency considerations).

The ability to evaluate several functions synchronously is very natural to define in the ideal model: the players simultaneously give all their inputs to the trusted party, who then gives them all the outputs (i.e., no output is given before all inputs are presented). We can also naturally define the corresponding semi-ideal model, where the players can ideally and simultaneously (i.e., within a single round) evaluate several functions. Assume now that we have a semiideal protocol $F$ for some function $f$ which simultaneously evaluates functions $g^{1}, \ldots, g^{k}$, and let $G_{i}$ be a secure protocol for $g^{i}$. Given an interleaving $I$ of the $G_{i}$ 's, we let $F^{I}$ denote the (real-model) protocol where we substitute the 
single ideal call to $g^{1}, \ldots, g^{k}$ with $k$ real executions of the protocols $G_{i}$ interleaved according to $I$. As apparent from Example 2, we cannot hope that every interleaving $I$ will be "good," that is, will yield a SFE protocol $F^{I}$ for $f$. (For instance, in the semi-ideal coin-flipping protocol $F$ of Example 2, no matter how we design SFE protocols $G_{1}$ and $G_{2}$ for $g^{1}$ and $g^{2}$, any sequential interleaving of $G_{1}$ and $G_{2}$ yields an insecure protocol.) Actually, the guaranteed existence of even a single good interleaving cannot be taken for granted, therefore:

Can we be guaranteed that there is always an interleaving $I$ of $G_{1}, \ldots, G_{k}$ such that $F^{I}$ is a SFE protocol for $f$ ?

Of course, the answer to the above question should depend on the notion of SFE we are using. This leads us to the following informal definition.

Definition 2: We say that a SFE notion satisfies synchronous reducibility if, whenever the protocols $F, G_{1}, \ldots, G_{k}$ satisfy this SFE notion, there exists an interleaving $I$ such that $F^{I}$ is a SFE protocol under this notion.

Example 2 not only shows that there are bad interleavings, but also that a "liberal" enough definition of SFE will not satisfy synchronous reducibility. In particular,

Lemma 2: The SFE notions of 5211 do not support synchronous reducibility.

Indeed, according to the SFE notions of 5211 , the protocol $G_{1}$ consisting of player $P_{1}$ sending $x_{1}$ to player $P_{2}$ is a secure protocol for $g^{1}$. Similarly, the protocol $G_{2}$ consisting of player $P_{2}$ sending $x_{2}$ to player $P_{1}$ is a secure protocol for $g^{2}$. However, there is no interleaving of $G_{1}$ and $G_{2}$ that will result in a secure coin-flip. This is because the last player to send its bit (which includes the case when the players exchange their bits in one round, due to the "rushing" ability of the adversary; see Section [2) is completely controlling the outcome.

On the positive side, we show 3

Theorem 2: A slight modification of the SFE notion of Micali and Rogaway [15] satisfies synchronous reducibility.

Theorem 2 actually has quite a constructive form. Namely, the nature of the definition in [15] not only guarantees that "good" interleavings $I$ always exist, but also that there are many of them, that they are easy to find, and that some of them produce efficient protocols. We summarize the last property in the following corollary.

Corollary 2: With respect to a slightly modified definition of SFE of Micali and Rogaway [15], let $F$ be an ideal protocol for $f$ that simultaneously calls functions $g^{1}, \ldots, g^{k}$, and let $G_{i}$ be an $R_{i}$-round SFE protocol for $g^{i}$. Then there exists (an easy to find) interleaving $I$ of the $G_{i}$ 's, consisting of at most $2 \cdot \max \left(R_{1}, \ldots, R_{k}\right)$ rounds, such that $F^{I}$ is a SFE protocol for $f$.

${ }^{3}$ As is illustrated in Section 4.3, the above "natural" protocols $G_{1}$ and $G_{2}$ are indeed insecure according to the definition of 15 . 
In other words, irrespective of the number of sub-protocols, we can synchronously interleave them using at most twice as many rounds as the longest of them takes 4 Let us remark that, unlike Corollary 1 (that simply follows from the definition of concurrent reducibility), Corollary 2 crucially depends on the very notion of [15], as is discussed more in Section 4.3.

In Sum. We have (1) clarified the notion of parallel reducibility, (2) distilled two important flavors of it, (3) modified slightly the SFE notion of Micali and Rogaway, and (4) showed that there exist SFE notions (e.g., the modified notion of [15]) as well as general SFE protocols (e.g., the one of [4]) that satisfy (both forms of) parallel reducibility.

Enjoying (both forms of) parallel reducibility do not necessarily imply that the definition of [15] is "preferable" to others. If the protocol one is designing is simple enough or is unlikely to be composed in parallel with other protocols, other definitions are equally adequate (and may actually be simpler to use). However, understanding which SFE notions enjoy parallel reducibility is crucial in order to simplify the complex task of designing secure computation protocols.

\section{The (Modified) Micali-Rogaway Definition of SFE}

Consider a probabilistic function $f(\mathbf{x}, r)=\left(f_{1}(\mathbf{x}, r), \ldots, f_{n}(\mathbf{x}, r)\right)$ (where $\mathbf{x}=$ $\left.\left(x_{1}, \ldots, x_{n}\right)\right)$. We wish to define a protocol $F$ for computing $f$ that is secure against any adversary $A$ that is allowed to corrupt in a dynamic fashion up to $t$ (out of $n$ ) players 5

\subsection{Protocols and Adversaries}

Protocol: An $n$-party protocol $F$ is a tuple $(\hat{F}, L R, C R, \mathcal{I}, \mathcal{O}, f, t)$ where

- $\hat{F}$ is a collection of $n$ interactive probabilistic Turing machines that interact in synchronous rounds.

- $L R$ - the last round of $F$ (a fixed integer, for simplicity).

- $C R$ - the committal round (a fixed integer, for simplicity).

- $\mathcal{I}$ - the effective-input function, a function from strings to strings.

- $\mathcal{O}$ - the effective-output function, a function from strings to strings.

- $f$ - a probabilistic function (which $F$ is supposed to compute).

- $t$ - a positive integer less than $n$ (a bound on the number of players that may be corrupted).

4 We note that the factor of 2 is typically too pessimistic. As it will be clear from the precise statement of synchronous reducibility in Section 3 natural protocols $G_{i}$ (like the ones designed using a general paradigm of [4]) can be synchronously interleaved in $\max \left(R_{1}, \ldots, R_{k}\right)$ rounds.

5 More generally, one can have an adversary that can corrupt only certain "allowable" subsets of players. The collection of these allowable subsets is usually called the adversary structure. For simplicity purposes only, we consider threshold adversary structures, i.e. the ones containing all subsets of cardinality $t$ or less. We call any such adversary $t$-restricted. 
Adversary: An adversary $A$ is a probabilistic algorithm.

Executing $\boldsymbol{F}$ and $\boldsymbol{A}$ : Adversary $A$ interacts with protocol $F$ as a traditional adaptive adversary in the rushing model. Roughly, this is explained below.

The execution of $F$ with an adversary $A$ proceeds as follows. Initially, each player $j$ has an input $x_{j}$ (for $f$ ) and an auxiliary input $a_{j}$, while $A$ has an auxiliary input $\alpha$. (Auxiliary inputs represent any a-priori information known to the corresponding party like the history of previous protocol executions. An honest player $j$ should ignore $a_{j}$, but $a_{j}$ might be useful later to the adversary.) At any point during the execution of $F, A$ is allowed to corrupt some player $j$ (as long as $A$ corrupts no more than $t$ players overall). By doing so, $A$ learns the entire view of $j$ (i.e., $x_{j}, a_{j}, j$ 's random tape, and all the messages sent and received by $j$ ) up to this point input. From now on, $A$ can completely control the behavior of $j$ and thus make $j$ deviate from $F$ in any malicious way. At the beginning of each round, $A$ first learns all the messages sent from currently good players to the corrupted ones 6 Then $A$ can adaptively corrupt several players, and only then does he send the messages from bad players to good ones. Without loss of generality, $A$ never sends a message from a bad player to another bad player.

At the end of $F$, the view of $A$, denoted $\operatorname{View}(A, F)$ consists of $\alpha, A$ 's random coins and the views of all the corrupted players. The traffic of a player $j$ up to round $R$ consists of all the messages received and sent by $j$ up to round $R$. Such traffic is denoted traffic $c_{j}(R)$ (or by $\operatorname{traffic}_{j}(R, F[A]$ ) whenever we wish to stress the protocol and the adversary executing with it).

Effective Inputs and Outputs of a Real Execution: In an execution of $F$ with $A$, the effective input of player $j$ (whether good or bad), denoted $\hat{x}_{j}^{F}$, is determined at the committal round $C R$ by evaluating the effective-input function $\mathcal{I}$ on $j$ 's traffic at round $C R: \hat{x}_{j}^{F}=\mathcal{I}\left(\right.$ traffic $\left._{j}(C R, F[A])\right)$. The effective output of player $j$, denoted $\hat{y}_{j}^{F}$, is determined from $j$ 's traffic at the last round $L R$ via the effective output function $\mathcal{O}: \hat{y}_{j}^{F}=\mathcal{O}\left(\operatorname{traffic}_{j}(L R, F[A])\right)$. Note that, for now, the effective inputs $\hat{\mathbf{x}}^{F}$ and outputs $\hat{\mathbf{y}}^{F}$ are unrelated to computing $f$.

History of a Real Execution: We let the history of a real execution, denoted $\operatorname{History}(A, F)$, to be $\left\langle\operatorname{View}(A, F), \hat{\mathbf{x}}^{F}, \hat{\mathbf{y}}^{F}\right\rangle$. Intuitively, the history contains all the relevant information of what happened when $A$ attacked the protocol $F$ : the view of $A$, i.e. what he "learned", and the effective inputs and outputs of all the players.

\subsection{Simulators and Adversaries}

Simulator: A simulator is a probabilistic, oracle-calling, algorithm $S$.

\footnotetext{
${ }^{6}$ We can even let the adversary schedule the delivery of good-to-bad messages and let him adaptively corrupt a new player in the middle of this process. For simplicity, we stick to our version.
} 
Executing $\boldsymbol{S}$ with $\boldsymbol{A}$ : Let $A$ be an adversary for a protocol $F$ for function $f$. In an execution of $S$ with $A$, there are no real players and there is no real network. Instead, $S$ interacts with $A$ in a round-by-round fashion, playing the role of all currently good players in an execution of $A$ with the real network, i.e.: (1) (makes up and) sends to $A$ a view of a player $j$ immediately after $A$ corrupts $j,(2)$ sends to $A$ the messages of currently good players to currently bad player: 7 and (3) receives the messages sent by $A$ (on behalf of the corrupted players) to currently good players. In performing these tasks, $S$ makes use of the following oracle $O(\mathbf{x}, \mathbf{a})$ :

- Before $C R$. When a player $j$ is corrupted by $A$ before the committal round, $O$ immediately sends $S$ the input values $x_{j}$ and $a_{j}$. In particular, $S$ uses these values in making up the view of $j$.

- At $C R$. At the end of the committal round $C R, S$ sends $O$ the value $\hat{x}_{j}^{S}=\mathcal{I}\left(\right.$ traffic $\left._{j}(C R)\right)$ for each corrupted player $j 9$ In response, $O$ randomly selects a string $r$, sets $\hat{x}_{j}^{S}=x_{j}$ for all currently good players $j$, computes $\hat{\mathbf{y}}^{S}=f\left(\hat{\mathbf{x}}^{S}, r\right)$, and for each corrupted player $j$ sends $\hat{y}_{j}^{S}$ back to $S$.

- After $C R$. When a player $j$ is corrupted by $A$ after the committal round, $O$ immediately sends $S$ the input values $x_{j}$ and $a_{j}$, as well as the computed value $\hat{y}_{j}^{S}$. In particular, $S$ uses these values in making up the view of $j$.

We denote by $\operatorname{View}(A, S)$ the view of $A$ when interacting with $S$ (using $O$ ).

Effective Inputs and Outputs of a Simulated Execution: Consider an execution of $S$ (using oracle $O(\mathbf{x}, \mathbf{a})$ ) with an adversary $A$. Then, the effective inputs of this execution consist of the above defined values $\mathbf{x}^{S}$. Namely, if a player $j$ is corrupted before the committal round $C R$, then its effective input is $\hat{x}_{j}^{S}=\mathcal{I}\left(\right.$ traffic $\left._{j}(C R, S[A])\right)$; otherwise ( $j$ is never corrupted, or is corrupted after the committal round) its effective input is $\hat{x}_{j}^{S}=x_{j}$. The effective outputs are the values $\mathbf{y}^{S}$ defined above. Namely, $\hat{\mathbf{y}}^{S}=f\left(\hat{\mathbf{x}}^{S}, r\right)$, where $r$ is the random string chosen by $O$ right after the committal round.

History of a Simulated Execution: We let the history of a simulated execution, denoted History $(A, S)$, to be $\left\langle\operatorname{View}(A, S), \hat{\mathbf{x}}^{S}, \hat{\mathbf{y}}^{S}\right\rangle$. Intuitively, the history contains all the relevant information of what happened when $A$ was communicating with $S$ (and $O$ ): the view of $A$, i.e. what he "learned", and the effective inputs and outputs of all the players.

\footnotetext{
$\overline{7}$ Notice that $S$ does not (and cannot) produce the messages from good players to good players.

${ }^{8}$ Such oracle is meant to represent the trusted party in an ideal evaluation of $f$. Given this oracle, $S$ 's goal is making $A$ believe that it is executing $F$ in a real network in which the players have inputs $\mathbf{x}$ and auxiliary inputs $\mathbf{a}$.

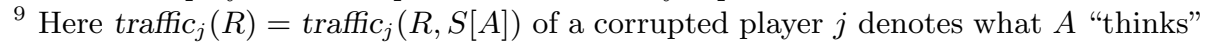
the traffic of $j$ after round $R$ is.
} 


\subsection{Secure Computation}

Definition 3: An $n$-party protocol $F$ is a SFE protocol for a probabilistic $n$ input/ $n$-output function $f(\mathbf{x}, r)$, if there exists a simulator $S$ such that for any input $\mathbf{x}=\left(x_{1}, \ldots, x_{n}\right)$, auxiliary input $\mathbf{a}=\left(a_{1}, \ldots, a_{n}\right)$, and any adversary $A$ with some auxiliary input $\alpha$, the histories of the real and the simulated executions are identically distributed:

$$
\operatorname{History}(A, F) \equiv \operatorname{History}(A, S)
$$

Equivalently, $\left\langle\operatorname{View}(A, F), \hat{\mathbf{x}}^{F}, \hat{\mathbf{y}}^{F}\right\rangle \equiv\left\langle\operatorname{View}(A, S), \hat{\mathbf{x}}^{S}, \hat{\mathbf{y}}^{S}\right\rangle$.

\subsection{Remarks}

Let us provide a minimal discussion of the above definition of SFE.

Simulators and Oracles vs. Ideal Adversaries. A standard benchmark in determining if a SFE notion is "reasonable" is the fact that for every real adversary $A$ there exists an "ideal adversary" $A^{\prime}$ that can produce (in the ideal model with the trusted party) the same view as $A$ got from the real network 10 We argue that the existence of a simulator $S$ in Definition 3 indeed implies the existence of such an adversary $A^{\prime} . A^{\prime}$ simply runs $A$ against the simulator $S$. If $A$ corrupts a player $j$ before the committal round, $A^{\prime}$ corrupts $j$ in the ideal model, and gives the values $x_{j}$ and $a_{j}$ (that it just learned) to $S$ on behalf of the oracle $O$. Right after the committal round of $F$ has been simulated by $S, A^{\prime}$ computes from the traffic of $A$ the effective inputs $\hat{x}_{j}^{S}$ of currently corrupted players $j$, hands them to the trusted party, and returns the outputs of the corrupted players to $S$ on behalf of $O$. Finally, if $A$ corrupts a player $j$ after the committal round, $A^{\prime}$ corrupts $j$ in the ideal model, and gives the values $x_{j}, a_{j}$ and the output of $j$ (that it just learned) to $S$ on behalf of the oracle $O$. At the end, $A^{\prime}$ simply outputs the resulting view of $A$ in the simulation 11

We notice, however, that the "equivalent" ideal adversary $A^{\prime}$ implied by our definition is much more special than the possible ideal adversary envisaged by other definitions (e.g., [5]) 12

\section{Our Modifications of the Original SFE Notion of Micali and Rogaway.}

We contribute a slightly cleaner and more powerful version of the SFE notion of [15]. Their original original notion was the first to advocate and highlight the importance of blending together privacy and correctness, a feature inherited by all subsequent SFE notions. We actually use a stronger (and more compactly expressed) such blending by demanding the equality of the joint distributions

${ }^{10}$ In fact, this requirement is more or less the SFE definition of [5].

11 The construction of $A^{\prime}$ intuitively explains the definition of effective inputs $\hat{\mathbf{x}}^{S}$ and effective outputs $\hat{\mathbf{y}}^{S}$ of the simulated execution, as they are exactly the inputs/outputs in the run of $A^{\prime}$ in the ideal model.

${ }^{12}$ For instance, such $A^{\prime}$ is constrained to run $A$ only once and in a black-box manner. 
of "view, inputs and outputs" in the real and in the simulated executions a suggestion of [11, which was followed by other SFE notions as well. We also extend the original SFE notion of [15] to include probabilistic functions.

Simulator Complexity. Because we are in an information-theoretic setting, we certainly do not want to impose any computational restrictions on the adversary. However, even though we chose not to do it for simplicity, we could demand that the simulator to be efficient (i.e., polynomial-time in the running time of the protoco 13 ). Indeed, (1) the natural simulator for the general protocol of [4] is efficient, and (2) our parallel-reducibility theorems would hold even if we required simulators to be efficient.

\section{The Notion of Parallel Reducibility}

First, let us define the semi-ideal model which generalizes the real model with the ability to ideally evaluate some functions. More precisely, in addition to regular rounds (where each player sends messages to other players), the semiideal model allows players to have ideal rounds. In such a round, the players can simultaneously evaluate several functions $g^{1}, \ldots, g^{k}$ using a trusted third party. More specifically, at the beginning of this round each player gives the $k$-tuple of his inputs to a trusted party. At the end of the round, each player gets back from the trusted party the corresponding $k$-tuple of outputs. (Note, these $k$-tuples are parts of players' traffic.)

Our definition of security of a protocol $F$ in the semi-ideal model is the same as that of a real model protocol with the following addition:

- The simulator $S$ has to simulate all the ideal rounds as well, since they are part of what the adversary $A$ expects. $S$ has to do this using no special " $g$ oracle". In other words, given the $g$-inputs of corrupted players in an ideal round, $S$ has to generate the corresponding outputs of corrupted players and give them back to $A$. Also, when $A$ corrupts a player $j, S$ has to produce on its own the $g$-inputs/outputs of player $j$ during all the ideal rounds that happened so far (as these are parts of $j$ 's traffic, and therefore $j$ 's view).

Let $F$ be a SFE protocol for $f$ in the semi-ideal model, and let us fix our attention on any particular ideal round $R$ that evaluates some functions $g^{1}, \ldots, g^{k}$. We say that the ideal round $R$ is order-independent if for any sequential ordering $\pi$ of $g^{1}, \ldots, g^{k}$, semi-ideal protocol $F$ remains secure if we replace the ideal round $R$ with $k$ ideal rounds evaluating a single $g^{i}$ at a time in the order given by $\pi$ (we denote this semi-ideal protocol by $F^{\pi}$ ).

${ }^{13}$ Some other SFE notions (e.g., that of [5]) demand that, for each adversary $A$, there is a simulator $S_{A}$ that is efficient compared to the running time of $A$. Note that such a requirement is meaningless in our definition. Indeed, our simulator is universal: it must reply "properly" and "on-line" to the messages it receives, without any knowledge of which adversary might have generated them. 
Let $G_{1}, \ldots, G_{k}$ be SFE protocols for $g^{1}, \ldots, g^{k}$. We would like to substitute the ideal calls to the $g^{i}$ 's with the corresponding protocols $G_{i}$ 's and still get a secure protocol for $f$. As we informally argued before, there are many ways to substitute (or to interleave) the $G_{i}$ 's, which is made precise by the following definition.

\section{Definition 4:}

- An interleaving of protocols $G_{1}, \ldots, G_{k}$ is any schedule $I$ of their execution. Namely, a single round of an interleaving may execute in parallel one round of one or more of the $G_{i}$ 's with the only restriction that the rounds of each $G_{i}$ are executed in the same order as they are in $G_{i}$.

- A synchronous interleaving of protocols $G_{1}, \ldots, G_{k}$ with committal rounds $C R_{1}, \ldots, C R_{k}$ is any interleaving $I$ such that for any $1 \leq i, \ell \leq k$, round $C R_{i}$ of $G_{i}$ strictly precedes round $C R_{\ell}+1$ of $G_{\ell}$. We call the place after all the "pre-committal" rounds but before all the "post-committal" rounds the synchronization point of $I$.

- Given an interleaving $I$ of $G_{1}, \ldots, G_{k}$, we let $F^{I}$ be a protocol obtained by substituting the ideal round $R$ with the execution of the protocols $G_{1}, \ldots, G_{k}$ in the order specified by $I$. The committal round of $F^{I}$, its effective input and output functions are defined in a straightforward manner from those of $F$ and $G_{1}, \ldots, G_{k}$. More specifically, given the traffic of player $j$ in $F^{I}$, we replace all $j$ 's traffic inside $G_{i}$ (if any) with the effective inputs and outputs of player $j$ in $G_{i}$, and apply the corresponding effective input/output function of $F$ to the resulting traffic. We also remark that when we run $G_{i}$, we let the auxiliary input of player $j$ to be its view of the computation so far.

The fundamental question addressed by parallel reducibility is

Assuming $F, G_{1}, \ldots, G_{k}$ are SFE protocols, under which conditions is $F^{I}$ a SFE protocol as well?

We highlight two kinds of sufficient conditions: (1) special properties of the protocol $F$ making $F^{I}$ secure irrespective of $I$ (which will lead us to concurrent reducibility), and (2) restrictions on the interleaving $I$ such that mere security of $F$ and $G_{1}, \ldots, G_{k}$ is enough (which will lead us to synchronous reducibility). The following Main Theorem restates Theorem 1 and 2 of the introduction.

Parallel-Reducibility Theorem: Consider the SFE notion of Definition 3. Let $F$ be a semi-ideal SFE protocol for $f$ evaluating $g^{1}, \ldots, g^{k}$ in an ideal round $R$; let $G_{i}$ be a SFE protocol for $g^{i}$; and let $I$ be an interleaving of $G_{1}, \ldots, G_{k}$. Then $F^{I}$ is a SFE protocol for $f$ if either of the following conditions holds:

1. (Concurrent-Reducibility Theorem) Round $R$ is order-independent.

2. (Synchronous-Reducibility Theorem) Interleaving $I$ is synchronous.

As we argued in the introduction, if we want $F^{I}$ to be secure for all $I$, round $R$ must be order-independent. Thus, the modified definition of Micali and Rogaway achieves the strongest form of concurrent reducibility. On the other, hand, we 
also argued that if we do not put any extra conditions on $F$ and $G_{1}, \ldots, G_{k}$ (aside from being SFE protocols), not all interleavings $I$ necessarily result in a SFE protocol. In fact, we showed in Lemma 2 that under a "too liberal" definition of SFE (which includes all SFE definitions other than Micali-Rogaway), it could be that no interleaving $I$ will result in a secure protocol $F^{I}$. The stringent definition of Micali-Rogaway (in particular, the existence of a committal round) not only shows that such an interleaving must exist, but also allows us to define a rich class of interleavings which guarantee the security of $F^{I}$ : the only thing we require is that all the "pre-committal" rounds precede all the "post-committal" rounds. In other words, players should first "declare" all their inputs to $g^{i}$ 's, and only then proceed with the "actual computation" of any of the $g^{i}$ 's. The intuition behind this restriction is clear: this is exactly what happens in the semi-ideal model when the players simultaneously evaluate $g^{1}, \ldots, g^{k}$ in $F$.

Remark 1: In the parallel-reducibility theorem we do not allow the adversary choose the interleaving $I$ adaptively in the process of the computation. This is only done for simplicity. For example, synchronous reducibility will hold provided the adversary is restricted to select a synchronous interleaving $I$. And concurrent reducibility holds if the semi-ideal protocol $F$ remains secure if we allow the semi-ideal adversary adaptively order the ideal calls to $g^{1}, \ldots, g^{k}$.

\section{Proof of the Parallel-Reducibility Theorem}

For economy and clarity of presentation, we shall prove both concurrent and synchronous reducibility "as together as possible". Let $S$ be the simulator for $F$, let $\pi$ be the order of committal rounds of the $G_{i}$ 's in the interleaving $I$ (if several committal rounds of $G_{i}$ 's happen in one round, order them arbitrarily), and let $S_{i}$ be the simulator for $G_{i}$. We need to construct the simulator $S^{I}$ for $F^{I}$. The proofs for the concurrent and synchronous reducibility are going to be very similar, the main differences being the following:

- Concurrent Reducibility. Since $R$ is an order-independent round of $F$, the protocol $F^{\pi}$ is also secure, i.e. has a simulator $S^{\pi}$. We will use $S^{\pi}$ instead of $S$ (together with $S_{1} \ldots S_{k}$ ) in constructing $S^{I}$. In particular, $S^{\pi}$ will simulate the ideal call to $g^{i}$ right after the committal round of $G_{i}$, which is exactly the order given by $\pi$.

- Synchronous Reducibility. Here we must use $S$ itself. In particular, at some point $S$ will have to simulate the simultaneous ideal call to $g^{1}, \ldots, g^{k}$, and expects to see all the inputs of the corrupted players. Since the interleaving $I$ is a synchronous interleaving, it has a synchronization point where all the effective inputs of the corrupted players are defined before any of the $G_{i}$ 's went on "with the rest of the computation." It is at this point where we let $S$ simulate the ideal call, because we will be able to provide $S$ with all the (effective) inputs.

To simplify matters, we can assume without loss of generality that each round of $I$ executes one round of a single $G_{i}$. Indeed, if we can construct a simulator for 
any such interleaving, we can do it for any interleaving executing in one round a round of several $G_{i}$ 's: arbitrarily split this round into several rounds executing a single $G_{i}$ and use the simulator for this new interleaving to simulate the original interleaving 14

\subsection{The Simulator $S^{I}$}

As we will see in Section 4.2, the actual proof will construct $S^{I}$ in $k$ stages, that is, will construct $k$ simulators $S^{1}, \ldots, S^{k}$, where $S^{k}$ will be $S^{I}$. However, we present the final $S^{I}$ right away because it provides a good intuition of why the proof "goes through".

For concreteness, we concentrate on the concurrent reducibility case. As one can expect, $S^{I}$ simply runs $S^{\pi}$ and uses $S_{1}, \ldots, S_{k}$ to simulate the interleaving of $G_{1}, \ldots, G_{k}$.

- Run $S^{\pi}$ up to round $R$ (can do it since $F^{I}$ and $F^{\pi}$ are the same up to round $R$ ).

- Tell each $S_{i}$ to corrupt all the players already corrupted by the adversary (it is irrelevant what we give to $S_{i}$ as their inputs).

- Assume we execute some round of protocol $G_{i}$ in the interleaving $I . S^{I}$ then uses $S_{i}$ to produce the needed messages from good-to-bad players and gives back to $S_{i}$ the response of the adversary.

- Right after the committal round $C R_{i}$ of $G_{i}$ has been simulated, use the effective input function of $G_{i}$ and the traffic of the adversary in the simulation of $G_{i}$ to determine the effective input $w_{j}^{i}$ of each corrupted player $j$ to $g^{i}$.

- We notice that at this stage $S^{\pi}$ is exactly waiting to simulate the ideal call to $g^{i}$ for the adversary. So $S^{I}$ gives $S^{\pi}$ the effective inputs $w_{j}^{i}$ as the adversary's inputs to $g^{i}$, and learns from $S^{\pi}$ the output $z_{j}^{i}$ of each corrupted player $j$.

- We notice that after round $C R_{i}$ has been simulated, the simulator $S_{i}$ expects to see the outputs of all the corrupted players from the $g^{i}$-oracle that does not exist in our simulation. Instead, we give $S_{i}$ the values $z_{j}^{i}$ that we just learned from $S^{\pi}$.

- We keep running the above simulation up to the end of the interleaving $I$. We note that at this stage $S^{\pi}$ has just finished simulating the ideal calls to all the $g^{i}$ 's, and waits to keep the simulation of $F^{\pi}$ starting from round $R+1$. And we just let $S^{\pi}$ do it intil the end of $F^{I}$ (we can do it since $F^{I}$ and $F^{\pi}$ are the same again from this stage).

- It remains to describe how $S^{I}$ handles the corruption requests of the adversary. This will depend on where in $F^{I}$ the corruption request happens. But in any case $S^{I}$ tells $S^{\pi}$ that the adversary asked to corrupt player $j$ and learns from $S^{\pi}$ the view $V_{j}$ of $j$ in (the simulation of) $F^{\pi}$.

${ }^{14}$ Here we use the fact that non-corrupted players execute all the $G_{i}$ 's independently from each other, so the adversary can only benefit by executing one round of a single $G_{i}$ at a time. 
* If the corruption request happens before round $R$, simply return $V_{j}$ to the adversary.

* Otherwise, the adversary expects to see (possibly partial) transcript of $j$ inside every $G_{i}$, which $V_{j}$ does not contain. However, $V_{j}$ still contains the supposed inputs $w_{j}^{i}$ of player $j$ to each $g^{i}$.

* For each $i$ we now ask the simulator $S_{i}$ to corrupt player $j$ in order to learn its view inside $G_{i}$. To answer this request, $S_{i}$ needs help from the $g^{i}$-oracle (that does not exist in our simulation), which $S^{I}$ provides as follows.

- If the corruption happened before the committal round $C R_{i}, S_{i}$ only expects to see the input and the auxiliary input of player $j$ to $g^{i}$. We give him $w_{j}^{i}$ as the actual input and extract from $V_{j}$ the view of $j$ prior to round $R$ as $j$ 's auxiliary input.

- If the corruption happened after round $C R_{i} 15 S_{i}$ also expects to see the output $z_{j}^{i}$ of player $j$ in $g^{i}$. However, in this case such an output is also contained in $V_{j}$, since right after the (already elapsed) round $C R_{i}$, we have simulated the ideal call to $g^{i}$ in $F^{\pi}$. Thus, $z_{j}^{i}$ is part of $j$ 's view in $F^{\pi}$, and as such should be included by $S^{\pi}$ in $V_{j}$.

* We see that in any of the above two cases we can provide $S_{i}$ with the information it expects. Therefore, we get back the view $W_{j}^{i}$ of $j$ in $G_{i}$ so far.

$\star S^{I}$ now simply combines $V_{j}$ with $W_{j}^{1}, \ldots, W_{j}^{k}$ to get the final simulated view of $j$, and gives it back to the adversary (we will argue later that the security of the $G_{i}$ 's implies that these views "match").

We remark that the simulator for synchronous reducibility is very similar. We essentially need to replace $S^{\pi}$ by $S$ and let $S$ simulate the single ideal call to $g^{1}, \ldots, g^{k}$ at the synchronization point of $I$, when the traffic of the adversary will simultaneously give $S$ the (effective) inputs of the corrupted players to all the $g^{i}$ s.

\subsection{Proof Outline}

While we have already constructed the simulator $S^{I}$, in the proof we will need to use the security of some particular $G_{i}$. Therefore, we will need "to move slowly" from the assumed secure protocol $F$ or $F^{\pi}$ (evaluating all the $g^{1}, \ldots, g^{k}$ ideally) to the protocol $F^{I}$ (whose security we need to establish and which runs $k$ real protocols $G_{1}, \ldots, G_{k}$ ). Roughly, we need to "eliminate" one ideal call (to some $g^{i}$ ) at a time, by "replacing" it with the protocol $G_{i}$. Using the security of $G_{i}$, we

$\overline{15}$ This includes the case when the corruption happened "after the end" of $G_{i}$. We treat this corruption as having the adversary corrupt player $j$ at the very end of the computation of $G_{i}$. This kind of "post-executuion" corruption has caused a lot of problems preventing some other SFE notions to satisfy reducibility. In our situation, this case presents no special problems due to the universality of the simulator and the information-theoretic security. 
will then argue that this "substitution" still leaves the resulting protocol a SFE protocol for $f$. To make the above idea more precise, we need some notation 16

First, from the interleaving $I$ of $G_{1}, \ldots, G_{k}$, we define the "projection interleaving" $I^{i}$ (for each $i \leq k$ ). This is the interleaving of the protocols $G_{1}, \ldots, G_{i}$ intermixed with the ideal calls to $g^{i+1}, \ldots, g^{k}$. More precisely, we remove from $I$ the rounds of all $G_{\ell}$ for $\ell>i$. For concurrent reducibility, we add the ideal calls to $g^{\ell}$ (for every $\ell>i$ ) right after the place where we previously had the committal round of $G_{\ell}$. We notice that this order of the ideal calls is consistent with the permutation $\pi$. In particular, we will identify the "base" interleaving $I^{0}$ of $g^{1}, \ldots, g^{k}$ with the permutation $\pi$. For synchronous reducibility, we add a single ideal call to $g^{i+1}, \ldots, g^{k}$ right at the synchronization point of $I$, and still call the resulting interleaving $I^{i}$ of $G_{1}, \ldots, G_{i}, g^{i+1}, \ldots, g^{k}$ a synchronous interleaving. Notice that $I^{i-1}$ is also a "projection" of $I^{i}$.

Slightly abusing the notation, we now define (in a straighforward way) "intermediate" semi-ideal protocols $F^{i}=F^{I^{i}}$, which essentially replace the ideal calls to $g^{1}, \ldots, g^{i}$ with $G_{1}, \ldots, G_{i}$ (but leave the ideal calls to $g^{i+1}, \ldots, g^{k}$ ). We note that $F^{k}=F^{I}$ and $F^{0}$ is either $F^{\pi}$ (the concurrent case) or $F$ (the synchronous case). We know by the assumption of the theorem that $F^{0}$ is secure, and need to show that $F^{k}$ is secure. Naturally, we show it by induction by showing that the security of $F^{i-1}$ implies that of $F^{i}$. Not surprisingly, this inductive step will follow from the security of $G_{i}$.

To summarize, the only thing we need to establish is the following. Assume $F^{i-1}$ is a SFE protocol for $f$ with the simulator $S^{i-1}$. We need to construct a simulator $S^{i}$ for $F^{i}$ such that for all inputs of the players and for any adversary $A^{i}$ in $F^{i}$, we get

$$
\operatorname{History}\left(A^{i}, F^{i}\right) \equiv \operatorname{History}\left(A^{i}, S^{i}\right)
$$

We construct $S^{i}$ from $S^{i-1}$ and the simulator $S_{i}$ for $G_{i}$. Essentially, $S^{i}$ will run $S^{i-1}$ in $F^{i}$ and use $S_{i}$ (together with $S^{i-1}$ 's simulation of the ideal call to $g^{i}$ ) to answer the adversary inside the $G_{i}$. In the "other direction", given adversary $A^{i}$ in $F^{i}$, we define the adversary $A^{i-1}$ in $F^{i-1}$. This adversary will run $A^{i}$ in $F^{i-1}$, and will also use $S_{i}$ (together with the ideal call to $g^{i}$ in $F^{i-1}$ ) to interact with $A^{i}$ inside $G_{i}$. Informally, we will say that " $S^{i}=S^{i-1}+S_{i}$ " and " $A^{i-1}=A^{i}+S_{i}$ ".

We observe that the security of $F^{i-1}$ implies that

$$
\operatorname{History}\left(A^{i-1}, F^{i-1}\right) \equiv \operatorname{History}\left(A^{i-1}, S^{i-1}\right)
$$

which is the same as

$$
\left\langle\operatorname{View}\left(A^{i-1}, F^{i-1}\right), \hat{\mathbf{x}}^{F^{i-1}}, \hat{\mathbf{y}}^{F^{i-1}}\right\rangle \equiv\left\langle\operatorname{View}\left(A^{i-1}, S^{i-1}\right), \hat{\mathbf{x}}^{S^{i-1}}, \hat{\mathbf{y}}^{S^{i-1}}\right\rangle
$$

Now, since $A^{i-1}$ essentially runs $A^{i}$ in the background, the view of $A^{i-1}$ (against both $F^{i-1}$ and $S^{i-1}$ ) will naturally "contain" the view of $A^{i}$. We denote these

\footnotetext{
${ }^{16}$ Below, we will try to use superscripts when talking about the notions related to computing $f$, like $F^{i}, S^{i}, A^{i}$. And we will use subscripts for the notions related to computing some $g^{i}$, like $G_{i}, S_{i}, A_{i}$.
} 
views by $\operatorname{View}\left(A^{i}, F^{i-1}+S_{i}\right)$ and $\operatorname{View}\left(A^{i}, S^{i-1}+S_{i}\right)$, and let

$$
\begin{aligned}
& \operatorname{History}\left(A^{i}, F^{i-1}+S_{i}\right) \stackrel{\text { def }}{=}\left\langle\operatorname{View}\left(A^{i}, F^{i-1}+S_{i}\right), \hat{\mathbf{x}}^{F^{i-1}}, \hat{\mathbf{y}}^{F^{i-1}}\right\rangle \\
& \operatorname{History}\left(A^{i}, S^{i-1}+S_{i}\right) \stackrel{\text { def }}{=}\left\langle\operatorname{View}\left(A^{i}, S^{i-1}+S_{i}\right), \hat{\mathbf{x}}^{S^{i-1}}, \hat{\mathbf{y}}^{S^{i-1}}\right\rangle
\end{aligned}
$$

Thus, Equation (3) (i.e., assumed security of $F^{i-1}$ ) implies that

$$
\operatorname{History}\left(A^{i}, F^{i-1}+S_{i}\right) \equiv \operatorname{History}\left(A^{i}, S^{i-1}+S_{i}\right)
$$

However, from the definition of $S^{i}=S^{i-1}+S_{i}$ and the definitions of the effective inputs/outputs of $F^{i}$ based on those of $F^{i-1}$, it will immediately follow that the latter distribution is syntactically the same as History $\left(A^{i}, S^{i}\right)$ ! That is,

$$
\operatorname{History}\left(A^{i}, S^{i-1}+S_{i}\right) \equiv \operatorname{History}\left(A^{i}, S^{i}\right)
$$

Therefore, Equation (77) and Equation (8) imply that what remains to prove in order to show Equation (2) is that

$$
\operatorname{History}\left(A^{i}, F^{i}\right) \equiv \operatorname{History}\left(A^{i}, F^{i-1}+S_{i}\right)
$$

We remark that the "environments" $F^{i}$ and $F^{i-1}+S_{i}$ are identical except the former runs the actual protocol $G_{i}$, while the latter evaluates $g^{i}$ ideally and uses the simulator $S_{i}$ to deal with $A^{i}$ inside $G_{i}$. Not surprisingly, the last equality (whose verification is the main technical aspect of the proof) will follow from the security of $G_{i}$. Namely, assuming that the last equality is false, we will construct an adversary $A_{i}$ for $G_{i}$ such that $\operatorname{History}\left(A_{i}, G_{i}\right) \not \equiv \operatorname{History}\left(A_{i}, S_{i}\right)$, a contradiction. Roughly, $A_{i}$ will simulate the whole network of players in $F^{i}$ (both the adversary $A^{i}$ and the honest players!), except when executing $G_{i}$.

This completes a brief outline of the proof. Additional details can be found in [9].

\subsection{The Definitional Support of Parallel Reducibility}

Since at least synchronous reducibility provably does not hold for other SFE definitions, one may wonder what specific features of our modified definition of [15] are "responsible" for parallel reducibility. While such key features can be properly appreciated only from the full proof of the parallel-reducibility theorem, we can already informally highlight two such features on the basis of the above proof outline.

On-Line Simulatability. The simulator $S$ not only is universal (i.e., independent of the adversary $A$ ) and not only interacts with $A$ in a black-box manner, but must also interact with $A$ "on-line". In other words, $S$ runs with $A$ only once: each time that $S$ sends a piece of information to $A$, this piece becomes part of $A$ 's final view. This is in contrast with traditional simulators, which would be allowed to interact with $A$ arbitrarily many times, to "rewind" $A$ in the middle of an execution, and to produce any string they want as $A$ 's entire view. 
The ability to generate $A$ 's final view on-line is probably the most crucial for achieving any kind of parallel reducibility. For example, an adversary $A$ of the composed protocol might base it actions in sub-protocol $G_{1}$ depending on what it sees in sub-protocol $G_{2}$ and vice versa. Therefore, the resulting views of $A$ inside $G_{1}$ and $G_{2}$ are very inter-dependent. It thus appears crucial that, in order to simulate these inter-dependent views, the simulator $S_{i}$ for $G_{i}$ should be capable of extending $A$ 's view inside $G_{i}$ incrementally "in small pieces" (as it happens with $A$ 's view in the real execution) that should never "be taken back". If, instead, we were only guaranteed that the simulator could simulate the entire (as opposed to "piece-by-piece") view of $A$ in each of the $G_{i}$ 's separately, there is no reason to expect that these separate views would be as inter-dependent as $A$ can make them in the real model. As demonstrated in Section 4.1] on the other hand, having on-line "one-pass" simulation makes it very easy to define the needed on-line simulator for $A$.

Committal Rounds. Intuitively, the committal round corresponds to the "synchronization point" in the ideal function evaluation: when all the players have sent their inputs to the trusted party, but have not received their corresponding outputs yet. Not surprisingly, the notion of the committal round plays such a crucial role in synchronous reducibility. In particular, the very existence of "good" interleavings (i.e., synchronous interleaving, as stated in Theorem 2) is based on the committal rounds. Committal rounds also play a crucial role in Corollary 2. Indeed, the greedy concurrent execution of all the "pre-committal" rounds of any number of sub-protocols $G_{1}, \ldots, G_{k}$ (which takes at $\operatorname{most} \max \left(R_{1}, \ldots, R_{k}\right.$ ) rounds), followed by the greedy concurrent execution of all the "post-committal" rounds of $G_{1}, \ldots, G_{k}$ (which also takes at most $\max \left(R_{1}, \ldots, R_{k}\right)$ rounds), yields a synchronous interleaving of $G_{1}, \ldots, G_{k}$ with the claimed number of rounds.

The Price of Parallel Reducibility. The definitional support of parallel reducibility "comes at a price": it rules out some reasonable protocols from being called secure. For example, having $P_{1}$ simply send $x_{1}$ to $P_{2}$ is not a secure protocol (in the sense of [15] and Definition 3) for the function $g^{1}\left(x_{1}, \lambda, \lambda, \ldots, \lambda\right)=$ $\left(x_{1}, x_{1}, \lambda, \ldots, \lambda\right)$ of Example 2. Indeed, assume the adversary $A$ corrupts player $P_{2}$ before the protocol starts and does not corrupt anyone else later on. Then $A$ will learn $x_{1}$ in the real execution. Therefore, for the simulator $S$ to match the view of $A$, it must also send $x_{1}$ to $A$ in round 1 . For doing so, $S$ must learn $x_{1}$ from its oracle before round 1 . Since $A$ does not corrupt player 1 , this can only happen when $S$ learns the output of corrupted player $P_{2}$ (which is indeed $x_{1}$ ) after the committal round. Unfortunately, the committal round is round 1 itself, because only then does $P_{1}$ manifest its input $x_{1}$ via its own message traffic. Thus, $S$ will learn $x_{1}$ only after round 1 , which is too late.

In sum, a reasonable protocol for function $g^{1}$ is excluded by the Definition 3 from being secure, but this "price" has a reason: Example 2 proves that such (individually) reasonable protocol is not synchronously reducible.

Parallel Reducibility in Other Settings. We have examined the concept of parallel reducibility in the information-theoretic setting. In particular, our proof 
of the parallel-reducibility theorem strongly uses information-theoretic security. It is a very interesting open question to examine parallel reducibility in the statistical and computational settings.

\section{References}

1. D. Beaver, Foundations of Secure Interactive Computing. Proc. of CRYPTO'91, pp. 377-391, 1991.

2. D. Beaver, Secure multi-party protocols and zero-knowledge proof systems tolerating a faulty majority. Journal of Cryptology, 4(2), pp. 75-122, 1991.

3. D. Beaver and S. Goldwasser, Multi-party computation with faulty majority, Proc. of the 30th FOCS, pp. 468-473, 1989.

4. M. Ben-Or, S. Goldwasser and A. Wigderson, Completeness Theorems for NonCryptographic Fault-Tolerant Distributed Computation, Proc. of the 20th STOC, pp. 1-10, 1998.

5. R. Canetti, Security and Composition of Multi-party Cryptographic Protocols. Journal of Cryptology, 13(1):143-202.

6. R. Canetti, Studies in Secure Multi-party Computation and Application, Ph.D. Thesis, Weizmann Institute, Israel, 1995.

7. D. Chaum, C. Crépeau and I. Damgård, Multiparty unconditionally secure protocols, Proc. of the 20th STOC, pp. 11-19, 1988.

8. R. Cramer, U. Maurer, and I. Damgård, General secure multiparty computation from any linear secret-sharing scheme, Proc. EUROCRYPT'00, pp. 316-334, 2000.

9. Y. Dodis and S. Micali. Parallel Reducibility for Information-Theoretically Secure Computation. Manuscript in progress.

10. P. Feldman and S. Micali, Optimal algorithms for Byzantine agreement, SIAM J. on Computing, 26(4):873-933, 1997.

11. S. Goldwasser and L. Levin, Fair computation of general functions in presence of immoral majority, Proc. CRYPTO '90, pp. 75-84, 1990.

12. O. Goldreich, Secure Multi-Party Computation, First draft available at http://theory.lcs.mit.edu/ oded.

13. O. Goldreich, S. Micali and A. Wigderson, How to play any mental game, Proc. of the 19th STOC, pp. 218-229, 1987.

14. K. Kilian, E. Kushilevitz, S. Micali and R. Ostrovsky, Reducibility and Completeness in Private Computations, To appear in SIAM J. on Computing, preliminary versions in Proc. of the 23rd STOC, 1991 by Kilian and in Proc. of the 35th FOCS, 1994 by Kushilevitz, Micali and Ostrovsky.

15. S. Micali and P. Rogaway, Secure computation, Proc. CRYPTO '91, pp. 392-404, 1991. Also in Workshop On Multi-Party Secure Computation, Weizmann Institute, Israel, 1998.

16. T. Rabin and M. Ben-Or, Verifiable Secret Sharing and Multi-party Protocols with Honest Majority, Proc. of 21st STOC, pp. 75-83, 1989.

17. A. Yao, Protocols for secure computation, Proc. of the 23rd FOCS, pp. 160-164, 1982. 